\title{
Detecção Precoce de Alzheimer Usando Machine Learning
}

\author{
Nathalia Paiva \\ Departamento de Informática \\ Pontifícia Universidade Católica do Rio de Janeiro (PUC-Rio) \\ Rio de Janeiro \\ npaiva@outlook.com
}

\author{
Tatiana Escovedo \\ Departamento de Informática \\ Pontifícia Universidade Católica do Rio de Janeiro (PUC-Rio) \\ Rio de Janeiro \\ tatiana@inf.puc-rio.br
}

\begin{abstract}
Resumo-A Doença de Alzheimer é uma doença neurodegenerativa, sendo responsável por aproximadamente de $60 \%$ a 80\% dos casos de demência no mundo e, por ainda não ter cura, pode acabar com a vida ou família do indivíduo que apresenta seus sintomas. Seu diagnóstico é realizado através dos resultados de exames laboratoriais, testes cognitivos e exames de imagens, sendo que as ressonâncias magnéticas são o método mais preciso. Por ser uma doença sem cura, o ideal é que seja diagnosticada o mais previamente possível, para que os médicos busquem desacelerar sua evolução e o paciente consiga manter uma vida sem depender de outras pessoas. Sabendo que a ciência de dados tem se mostrado eficaz no apoio ao diagnóstico em diversas áreas da medicina, no presente trabalho testamos o desempenho de algoritmos inteligentes na classificação de características extraídas de imagens de ressonâncias magnéticas. Conseguimos uma acurácia de aproximadamente $73 \%$ ao prever o declínio cognitivo baseado em características extraídas do dataset OASIS-1, disponibilizado pelo Open Access Series of Imaging Studies, utilizando o algoritmo de regressão logística. Distintas configurações de classificadores foram utilizadas a fim de avaliá-los, tomando como base a maximização do desempenho e minimização custo computacional. Concluímos que é possível prever a doença utilizando características como idade, nível socioeconômico e nível de educação.
\end{abstract}

Palavras Chave-Alzheimer, Demência, OASIS-1, Machine Learning, Ciência de Dados

\footnotetext{
Abstract-Alzheimer's disease is a neurodegenerative disease, responsible for approximately $60 \%$ to $80 \%$ of cases of dementia in the world and, as it still has no cure, it can end the life or family of the individual who presents its symptoms. Its diagnosis is made through the results of laboratory exams, cognitive tests and image exams, and MRIs are the most accurate method. As it is a disease without cure, the ideal is that it is diagnosed as soon as possible, so that doctors can slow down its evolution and the patient can maintain a life without depending on other people. Knowing that data science has been shown to be effective in supporting diagnosis in several areas of medicine, that is why in the present work we tested the performance of intelligent algorithms in the classification of characteristics extracted from magnetic resonance images. We achieved an accuracy of approximately $73 \%$ by predicting cognitive decline based on characteristics extracted from the OASIS-1 dataset, made available by the Open Access Series of Imaging Studies, using the logistic regression algorithm. Different classifier configurations
}

were used in order to evaluate them, based on maximizing performance and minimizing computational cost. We concluded that it is possible to predict the disease using characteristics such as age, socioeconomic status and level of education.

Keywords-Alzheimer, Dementia, OASIS-1, Machine Learning, Data Science

\section{INTRODUCC̃̃O}

Segundo a Organização Mundial da Saúde (OMS), os casos de demência vão triplicar e chegar a 152 milhões de pessoas até 2050 [1]. Com o aumento da expectativa de vida, graças ao avanço da tecnologia médica que veio a oferecer bons feitos tecnológicos nas prevenções de doenças, tratamento, fármacos e ao maior acesso a serviços de saúde, o índice de crescimento de casos de demência tornou-se um dos principais desafios da saúde pública, com 40-50 milhões de pessoas que vivem atualmente com esta condição [2]. O cuidado a tais pacientes tem consequências amplas para as famílias, os sistemas de saúde e a sociedade como um todo. A demência engloba uma série de doenças progressivas que afetam as capacidades de atenção, memória e outras habilidades cognitivas e comportamentais [3]. Há mais de 100 formas de demência e a mais comum é a doença de Alzheimer, que contabiliza de $60 \%$ a $80 \%$ de todos os casos [4]. Na Figura 1 podemos ver a porcentagem de casos de outras formas de demência, como por corpos de Lewy, vascular e frontotemporal [5]. 


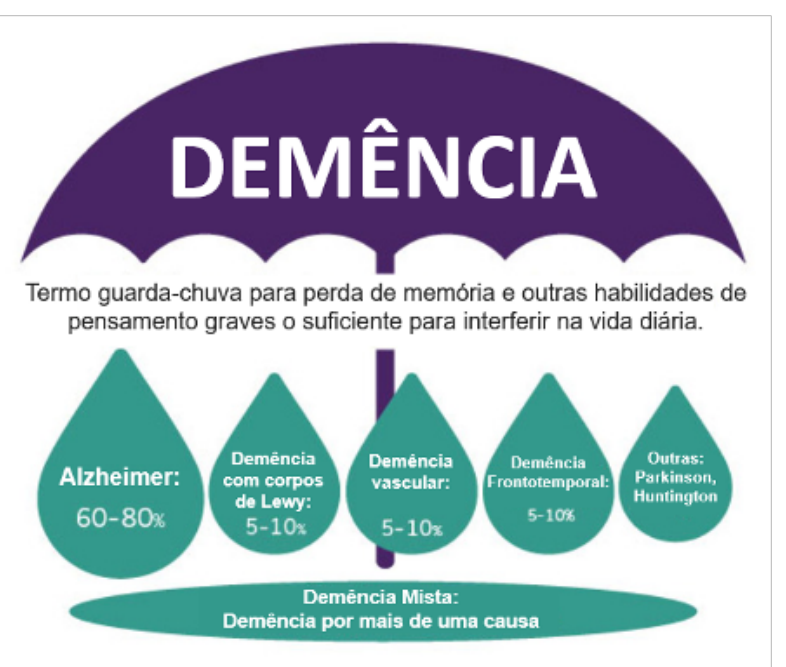

Fig. 1. Porcentagem de casos de demência. Fonte: adaptado de [5]

A demência é uma doença neurodegenerativa cujo desenvolvimento modifica estruturalmente o cérebro, especialmente nas regiões responsáveis por funções cognitivas. O cérebro humano cresce gradativamente até os 21 anos, a partir de então se dá início a um declínio progressivo, porém discreto. Já o volume cerebral apresenta um declínio apenas a partir dos 60 anos, o qual se acentua entre 70 e 90 anos. A atrofia anual do cérebro de um paciente com doença neurodegenerativa está entre 2 e $3 \%$, enquanto indivíduos saudáveis apresentam de 0.2 a $0.5 \%$ de atrofia [5].

As doenças neurodegenerativas apresentam quatro estágios clínicos, e inicialmente há perda de memória, mudança de personalidade e nas habilidades de locomoção. O estágio moderado é caracterizado pela dificuldade de fala e realização de atividades diárias, além de agitação e insônia. Quando no estágio avançado o indivíduo apresenta incontinência urinária e fecal, resistência à execução de tarefas diárias, dificuldades para comer e deficiência motora progressiva. Por fim, o estágio terminal caracteriza-se por perda total de memória e dependência de terceiros [6].

Atualmente não há cura para a demência e, por isso, quanto mais cedo o diagnóstico for feito, maiores serão as chances de tratar os sintomas corretamente, retardando a evolução da doença. Com o auxílio do exame de ressonância magnética, um bom médico é capaz de identificar os primeiros indícios da doença, ou para casos mais avançados, seu grau de severidade. O diagnóstico precoce é o ponto chave para iniciar o tratamento correto e, com o avanço da tecnologia, podemos utilizar técnicas de Machine Learning para apoiar o acompanhamento do progresso de um paciente, desde a fase de comprometimento cognitivo leve até a fase da demência.

Desta forma, este artigo visa verificar o desempenho de modelos de Machine Learning que possam ser usados para ajudar os médicos na detecção precoce da demência, utilizando como base características básicas do paciente. Para tal, será utilizando o dataset disponibilizado pelo Open Access Series of Imaging Studies (OASIS) [7], um projeto que visa disponibilizar gratuitamente os conjuntos de dados do cérebro para facilitar descobertas futuras em neurociência básica e clínica. O dataset escolhido é o OASIS-1, que possui dados de ressonância magnética.

$\mathrm{Na}$ Seção 2 deste artigo serão apresentados alguns projetos e pesquisas já realizados nessa área da saúde, que podem fazer uma grande diferença na vida de quem tem a doença, dando novas esperanças e expectativas de vida. Logo após, na Seção 3, comentamos um pouco sobre a história do Machine Learning, seus diferentes métodos e utilidades. Já na Seção 4, detalharemos o dataset escolhido para o experimento. $\mathrm{Na}$ Seção 5 serão detalhadas as manipulações de dados, algoritmos executados no dataset e resultados obtidos, e por fim, na Seção 6 serão apresentadas as conclusões do experimento.

\section{REVISÃO DA LITERATURA}

Na literatura, há inúmeros estudos sobre a demência, seja para descobrir sua causa, relação com outras doenças, possível reversão dos sintomas ou diagnóstico precoce. A seguir, são apresentados alguns dos estudos relacionados que podem agregar nosso conhecimento sobre a evolução da ciência no assunto, utilizando ou não ciência de dados.

\section{A. Inteligência artificial prevê Alzheimer anos antes do diagnóstico}

De acordo com um estudo publicado na Radiology [8], é possível usar a inteligência artificial (IA) para prever a doença de Alzheimer através da análise de imagens do cérebro. A pesquisa ligou $\mathrm{o}$ processo da doença a alterações no metabolismo, como mostrado pela captação de glicose em certas regiões do cérebro, porém essas alterações podem ser difíceis de reconhecer. "As diferenças no padrão de captação de glicose no cérebro são muito sutis e difusas", disse o coautor do estudo, Jae Ho Sohn, MD, do Departamento de Radiologia e Imagem Biomédica da Universidade da Califórnia em San Francisco (UCSF). "As pessoas são boas em encontrar biomarcadores específicos de doenças, mas as alterações metabólicas representam um processo mais global e sutil”.

Pesquisadores e cientistas obtiveram acesso dos dados da iniciativa Neuroimagem da Doença de Alzheimer (ADNI), o qual é considerado um importante estudo científico, focado em ensaios clínicos para melhorar a prevenção e o tratamento da doença. Com esse foco os cientistas treinaram um algoritmo de Deep Learning usando uma varredura de 18-Ffluorodeoxiglucosw PET (FDG_PET). O Fluorodesoxiglicose é um radiofármaco usado na tomografia por emissão de positrões, uma modalidade de imagiologia médica.

O conjunto de dados ADNI incluiu mais de 2.100 imagens cerebrais de FDG-PET de 1.002 pacientes, as quais foram divididas em $90 \%$ para o treinamento do algoritmo e $10 \%$ para teste. Em seguida, os pesquisadores testaram o algoritmo em um conjunto independente de 40 exames de imagem, de 40 pacientes que nunca haviam estudado e obtiveram $100 \%$ de sensibilidade na detecção de características da doença. Com isso, seria possível prever a doença em até 6 anos antes do paciente apresentar sintomas e ter o diagnóstico final.

\section{B. Cientistas encontram relação entre anemia e aumento no risco de demência}


De acordo com um estudo publicado na Neurology [9], cientistas concluíram que é possível ter uma relação direta entre o risco maior de desenvolver demência quando um adulto envelhece e seus níveis de hemoglobina estarem acima ou abaixo do normal.

A hemoglobina é uma proteína presente nos glóbulos vermelhos e a responsável pela coloração vermelha do sangue. Tem como função o transporte de oxigênio pelo corpo e quando há queda na concentração dos níveis considerados normais no sangue, causa a anemia [10].

O estudo foi realizado em certa de doze mil participantes, com idade média de 64 anos, ao longo de 12 anos. Ao final do tempo proposto, foi constatado que 1.520 indivíduos haviam desenvolvido demência. Observaram uma associação em forma de $U$ entre os níveis de hemoglobina e demência, de modo que tanto os níveis baixos quanto os altos foram associados com risco aumentado de demência.

Os cientistas explicaram que, como a hemoglobina transporta oxigênio pelo corpo, se houver muito pouco, algumas partes do cérebro podem ficar sem, podendo causar uma inflamação e danificar o cérebro. Quanto aos níveis altos de hemoglobina, a teoria é tornaria o sangue mais viscoso, dificultando sua entrada em vasos sanguíneos menores e reduzindo potencialmente o suprimento de oxigênio no cérebro.

\section{Reversão dos sintomas}

De acordo com o estudo publicado na ScienceDaily[11], é provável que seja possível reverter a Alzheimer em casos de estágio inicial.

Usando o Programa Terapêutico Pessoal de Saúde Afirmativa (PTPr), cientistas procuraram determinar se um programa abrangente e personalizado poderia melhorar a função cognitiva e metabólica em indivíduos com diagnóstico de deficiência cognitiva subjetiva e deficiência cognitiva leve.

Os pesquisadores tiveram acesso a amostras de sangue e pontuações da Avaliação Cognitiva de Montreal (MoCA) de 35 indivíduos. Eles então participaram de um programa de imersão de quatro dias, que incluiu Planos de Terapêutica Pessoal (PTP), consultas com médicos e explicações sobre o PTPr e PTP. Os participantes tiveram acompanhamento por telemonitoramento, com análises repetidas de amostras de sangue, atualizações sobre escolhas de estilo de vida, medicamentos e suplementos atuais e teste de MoCA pelo menos uma vez entre 3 e 12 meses após os 4 dias iniciais.

Com isso, ao comparar a linha de base com o teste de acompanhamento, determinaram vários pontos de fatores de risco, incluindo glicose no sangue e níveis de insulina. Os níveis de vitaminas B12, D3 e E, melhoraram em todo o grupo de participantes ou especificamente em indivíduos com medidas fora do normal. As pontuações do MoCA foram estabilizadas em todo o grupo de participantes e melhoraram significativamente em indivíduos com pontuação de $24 \mathrm{ou}$ menos no início do estudo.

Concluíram que os resultados fornecem evidências de que uma abordagem abrangente e personalizada projetada para mitigar os fatores de risco do Alzheimer pode melhorar pontos dos fatores de risco e estabilizar a função cognitiva, garantindo estudos clínicos mais extensos e controlados por placebo.

É possível perceber nos estudos citados que os cientistas estão procurando maneiras de prever, correlacionar ou até mesmo reverter sintomas desta doença neurodegenerativa, utilizando técnicas ou dados que não possuíam anteriormente. Este trabalho tem exatamente o mesmo objetivo, apesar de Machine Learning não ser um método atual, conseguimos provar que utilizando o algoritmo e métricas certas conseguimos diagnosticar ou até mesmo detectar a possibilidade de o indivíduo ter a doença em algum momento de sua vida.

\section{MACHINE LEARNING}

Como dito anteriormente, Machine Learning não é algo atual, foi o um termo criado em 1959 por Arthur Samuel, engenheiro do Massachusetts Institute of Technology (MIT), o qual descrevia o conceito como "um campo de estudo que dá aos computadores a habilidade de aprender sem terem sido programados para tal" [12].

Machine Learning é uma subárea da inteligência artificial, na qual é possível criar algoritmos ou utilizar os já existentes para ensinar uma determinada máquina a desempenhar tarefas. Utilizamos os algoritmos para coletar dados, aprender com eles, saber identificar os padrões e com isso fazer predições sobre situações similares com as que foram aprendidas [13]. Por exemplo, temos os filtros de spam nos e-mails, algoritmos foram treinados para identificar remetentes e/ou conteúdo dos e-mails suspeitos e assim classificá-los como spam ou confiáveis.

Há diferentes métodos de aprendizado, sendo os mais populares o aprendizado não supervisionado e supervisionado. O não supervisionado é usado em dados que o sistema não sabe qual será a saída correta. $\mathrm{O}$ algoritmo não recebe durante o treinamento quais os resultados esperados, então ele tenta descobrir, explorando os dados e encontrando possíveis padrões e/ou relacionamentos .Um exemplo de uso é na recomendação de filmes na Netflix.

Ao contrário do aprendizado não supervisionado, o supervisionado é aquele em que durante o treinamento o algoritmo recebe o conjunto de dados já rotulado, ou seja, os dados de treinamento já contêm as saídas esperadas. O algoritmo aprende os padrões para então quando receber dados novos, sem estarem rotulados, utilizar o que aprendeu anteriormente para fazer a classificação [13]. Como exemplo, podemos citar o tema deste trabalho, no qual temos o conjunto de dados entrada e sabemos que a saída será o nível de demência do indivíduo.

\section{ANÁLISE EXPLORATÓRIA DOS DADOS}

Conforme mencionado na introdução deste trabalho, usaremos para esse estudo o dataset OASIS-1, que apresenta dados de ressonância magnética de jovens e adultos, nãodementes e dementes. Todos os indivíduos do dataset são destros e são incluídos homens e mulheres. 100 dos indivíduos incluídos acima de 60 anos foram diagnosticados clinicamente com doença de Alzheimer, de muito leve a moderada, um total de $22,9 \%$ do dataset.

Nele há 436 linhas de dados extraídos de 41 imagens de ressonância magnética de indivíduos com idades entre 18 e 96 
anos. Para cada pessoa, há 3 ou 4 exames de ressonância magnética individuais ponderados em $\mathrm{T} 1$, onde há melhor visualização da gordura nas veias, obtidos em uma mesma sessão.

As ressonâncias magnéticas produzem imagens de cortes finos de tecidos (imagens tomográficas) utilizando um campo magnético e ondas de rádio. Softwares determinam como a imagem será ponderada e como os vários tecidos irão aparecer, usando as radiofrequências de pulso e das ondas de gradientes. As imagens ponderadas em T1 mostram a anatomia de tecidos moles e gordura, já as imagens ponderadas em T2 mostram, líquidos e patologias, como tumores, inflamação e trauma [14].

Por exemplo, a gordura aparece brilhante (alta intensidade de sinal) na imagem ponderada em $\mathrm{T} 1$ e relativamente escura (baixa intensidade de sinal) na imagem ponderada em T2, água e líquidos aparecem mais escuros na imagem ponderada em T1 e brilhantes nas imagens ponderada em T2. Na prática, as imagens ponderadas em T1 e T2 fornecem informações complementares, de forma que ambas são importantes para caracterizar a patologia. Como dito acima, nosso dataset possui apenas as informações de ressonâncias ponderadas em T1. A Figura 2 apresenta um exemplo da ressonância ponderada em $\mathrm{T} 1$ comparando com a ponderada em $\mathrm{T} 2$.

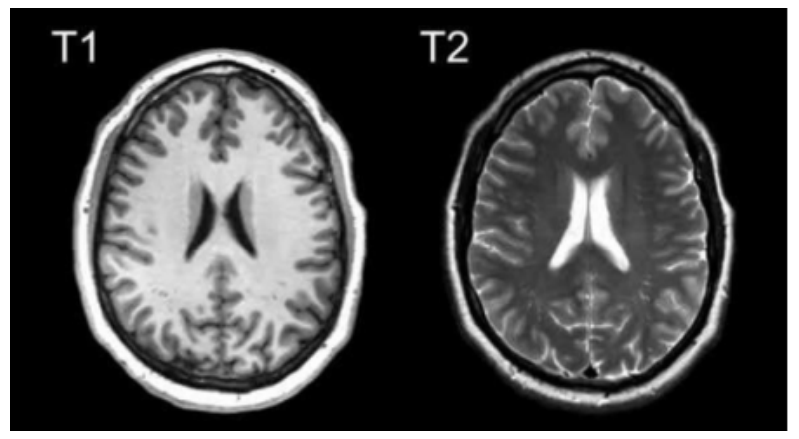

Fig. 2. Ressonâncias ponderadas em T1 e T2

A Tabela 1 descreve cada coluna do arquivo OASIS- 1 .

TABELA 1. DESCRIÇ̃̃O DOS CAMPOS DO DATASET

\begin{tabular}{|c|c|}
\hline NOME DA COLUNA & DESCRIÇÃO \\
\hline ID & Identificador \\
\hline M/F & Gênero masculino ou feminino \\
\hline Hand & Mão (R - Destro / L - Canhoto) \\
\hline Age & Idade \\
\hline Educ & Status socioeconômico de educac̃o \\
\hline SES & Mini exame do estado mental \\
\hline MMSE & Avaliação clínica de demência \\
\hline CDR & Volume intracraniano total estimado \\
\hline eTIV & Volume do cérebro inteiro normalizado \\
\hline nWBV & Fator de escala atlas \\
\hline ASF & Dias entre as visitas \\
\hline Delay &
\end{tabular}

Utilizando Jupyter notebook e Python, realizamos uma análise exploratória para conhecer um pouco mais sobre os dados, verificando suas informações, explorando o conteúdo de algumas linhas, sua dimensão, tipo dos atributos, média, desvio padrão e principalmente a quantidade de dados $\mathrm{NaN}$ (ausentes). Na maioria dos atributos não há muitos $\mathrm{NaN}$, mas há casos como Educ, SES e CDR, onde praticamente metade dos dados estão $\mathrm{NaN}$, podendo impactar as análises. A Tabela 2 mostra a quantidade de $\mathrm{NaN}$ em cada atributo.

TABELA 1. COLUNAS X CAMPO VAZIO

\begin{tabular}{|c|c|}
\hline COLUNAS & QUANTIDADE NaN \\
\hline ID & 0 \\
\hline M/F & 0 \\
\hline Hand & 0 \\
\hline Age & 0 \\
\hline Educ & 201 \\
\hline SES & 220 \\
\hline MMSE & 201 \\
\hline CDR & 201 \\
\hline eTIV & 0 \\
\hline nWBV & 0 \\
\hline ASF & 0 \\
\hline Delay & 416 \\
\hline
\end{tabular}

Por enquanto, sem realizamos nenhum tratamento no dataset, validamos a relação entre os campos usando a Correlação de Pearson. Quando o resultado se aproxima de 1 significa que há um aumento no valor de uma variável quando a outra também aumenta, ou seja, temos uma relação linear positiva. Quando o coeficiente se aproxima de -1, temos uma correlação negativa ou inversa, significando que quando o valor de uma variável aumenta o da outra diminui. Quando o coeficiente de correlação é próximo de zero, indica que não há relação entre as duas variáveis. Ou seja, quanto mais eles se aproximam de 1 ou -1 , mais forte é a relação. Na Figura 3, podemos visualizar o resultado da análise, na qual podemos concluir que há mais correlações negativa do que positivas.

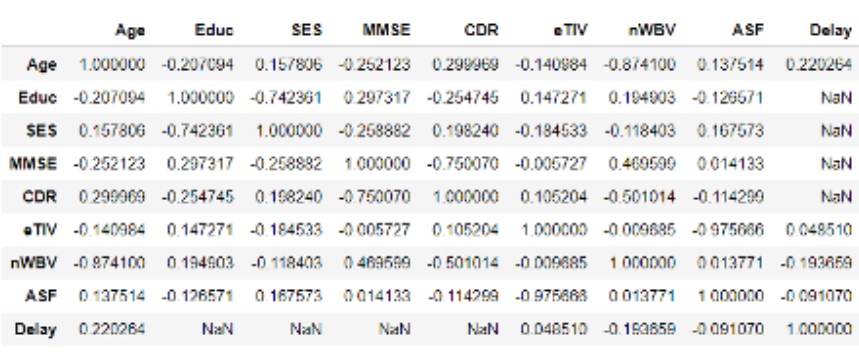

Fig. 3. Correlação de Pearson antes do tratamento dos dados

A idade e WBV, Educ e SES, MMSE e CDR, eTIV e ASF são exemplos de correlações negativas fortes, pois variam entre $-0,74$ e -0,97, já em relação a correlações positivas, o máximo que conseguimos é 0,46 entre MMSE e WBV, não indicando correlação significativa.

Mesmo sem nenhum tratamento prévio no dataset, podemos ver que as ligações fazem sentido. Por exemplo, pesquisas mostram que é normal o tamanho do cérebro alterar conforme a idade, independente se a pessoa sofre de alguma doença degenerativa ou não. 
O resultado da correspondência entre o nível de educação e o status socioeconômico mostra que quanto maior o nível socioeconômico, maior é o nível educacional.

Entre o mini exame do estado mental e a avaliação clínica de demência há de fato uma relação negativa. Apesar de ambos terem o mesmo intuito (classificar o nível de demência da pessoa), os resultados são opostos: no mini exame, quanto maior for a pontuação do paciente, mais saudável ele está e na avaliação clínica, quanto maior for a pontuação, maior é a gravidade da demência.

Por fim, a normalização do atlas equivale ao tamanho da cabeça, logo realmente ASF deve ser proporcional ao TIV. A correlação positiva que obtivemos também está correta, considerando que um cérebro não saudável é menor, um cérebro com tamanho normal tem uma pontuação maior no mini exame.

Após essas análises no dataset original, fizemos uma limpeza em alguns dados. Como todos os pacientes dessa amostra são destros, não temos como argumentar que haja alguma relação entre a mão com a qual a pessoa escreve e uma possível demência. Também não conseguimos utilizar o atributo delay como parâmetro, pois a grande maioria está como NaN. Logo, eliminamos esses dois atributos do nosso dataset.

Para efeito de análise, também foram removidas as linhas nas quais o CDR, SES e Educ eram NaN. Refizemos a correlação de Perason nessa nova versão do dataset para vermos o impacto da alteração e obtivemos o resultado ilustrado na Figura 4.

\begin{tabular}{|c|c|c|c|c|c|c|c|c|}
\hline & Age & Edue & SES & MMSE & CDR & - TIV & nWBV & ASF \\
\hline Age & 1.000000 & -0.206650 & 0.157806 & -0.310579 & 0.310571 & 0.038419 & -0.735933 & -0.034442 \\
\hline Educ & 0.206650 & 1.000000 & .0 .742361 & 0.298136 & 0.233157 & 0.166223 & 0.171876 & -0.143056 \\
\hline SES & 0.157808 & -0.742381 & 1.000000 & -0.258882 & 0.189240 & -0.184533 & -0.118403 & 0.167573 \\
\hline MMSE & .0 .310579 & 0.288136 & $-0.25 a 8 a 2$ & 1.000000 & 0.775791 & -0.010140 & 0.480587 & 0.019879 \\
\hline CDR & 0.310571 & -0.233157 & 0.198240 & -0.775791 & 1.000000 & 0.113593 & -0.499226 & -0.124387 \\
\hline eTIV & 0038419 & 0166223 & -0.184533 & -0010140 & 0.113580 & 1.000000 & -0214031 & -0989518 \\
\hline nWBV & -0.735933 & 0.171876 & -0.118403 & 0.480587 & -0.400226 & -0.214031 & 1.000000 & 0.217107 \\
\hline ASF & -0.034442 & -0.143058 & 0.187573 & 0019879 & -0.124387 & $-0.98951 a$ & 0217107 & 1.000000 \\
\hline
\end{tabular}

Fig. 4. Correlação de Pearson após tratamento dos dados

Comparando com o resultado anterior, observamos que não houve nenhuma alteração significativa, pois nenhum resultado alterou tanto a ponto de chegar mais próximo de -1 ou +1 .

\section{SOLUÇÃO PROPOSTA}

Utilizando as informações de Age, SES e Educ do novo dataset para conseguirmos determinar o valor de CDR, identificaremos a presença da doença partindo de informações básicas. Para isso, vamos avaliar 5 diferentes algoritmos de Machine Learning para classificação: Regressão Logística, Random Forest, Naive Bayes, Multilayer Perceptron e KNN.

A regressão logística é uma técnica estatística que tem como objetivo produzir um modelo que permita a predição de valores tomados por uma variável categórica, frequentemente binária, a partir de uma série de variáveis explicativas contínuas e/ou binárias [15]. Então, a partir desse modelo gerado é possível calcular ou prever a probabilidade de um evento ocorrer, dado uma observação aleatória.

O algoritmo Random Forest é criado por várias árvores de decisão, combinando a simplicidade das árvores com a flexibilidade e aleatoriedade para melhorar a precisão, com o objetivo de resolver o maior problema das arvores de decisão que é a propensão ao overfitting. O overfitting é o termo para descrever quando o algoritmo se ajusta muito bem a determinado conjunto de dados, mas não é capaz de fazer boas predições em um novo conjunto. Quando a árvore cresce bastante e não trabalhamos muito bem seu crescimento, ela pode se ajustar demais aos dados de treinamento, o Random Forest resolve esse problema escolhendo aleatoriamente os dados e criando diversas combinações simples de arvores com eles [16][17].

Naive Bayes é um classificador probabilístico, baseado no Teorema de Bayes. Ele calcula a probabilidade de o dado pertencer a uma classe baseado em suas características. O algoritmo divide o dataset por cada característica e calcula a probabilidade de cada uma pertencer a uma classe, então quando recebe um novo dado, ele utiliza esse cálculo para determinar a qual classe o novo dado pertence [18].

O algoritmo Multilayer Perceptron (MLP) é uma classe de rede neural artificial feedforward (ANN) que consiste em três ou mais camadas de nós, sendo uma camada de entrada, uma ou mais camadas ocultas e uma camada de saída. Exceto para os nós de entrada, cada nó é um neurônio que usa uma função de ativação não linear e todos estão conectados a sua camada anterior. MLP utiliza a técnica de aprendizado chamada backpropagation [19].

A técnica backpropagation possui duas fases, a propagação e a retropropagação, onde na propagação os pesos são calculados aleatoriamente e propagados por toda a árvore até que na camada final, é calculado o erro com base no valor esperado e dependendo do erro, na tentativa de diminui-lo é recalculado o valor dos pesos da última camada de neurônios, com base em seus gradientes, e assim repropaga para as camadas anteriores, essa é a retropropagação. Como o vetor gradiente fornece a direção de maior crescimento de uma função e queremos o menor crescimento da função de erro, tomamos o sentido contrário ao do gradiente e fazemos isso de forma iterativa, sempre recalculando os gradientes em cada passo de iteração, até o erro ser o mínimo possível [20].

$\mathrm{O}$ algoritmo KNN consiste em classificar o dado com base na classe de seus vizinhos. $\mathrm{O}$ dado é atribuído à classe mais comum entre seus $\mathrm{k}$ vizinhos mais próximos, sendo $\mathrm{k}$ um número inteiro positivo. Por exemplo, se $\mathrm{k}$ for igual a 1 , então o objeto é simplesmente atribuído à classe daquele único vizinho mais próximo, caso for igual à 3 , será atribuído a classe da maioria dos vizinhos [21].

Para avaliar o desempenho de cada algoritmo iremos analisar a acurácia dos resultados. A acurácia da classificação é definida como a porcentagem do número de amostras classificadas corretamente dividido pelo número total de amostras obtidas.

Para as análises separamos o dataset em dois grupos, de teste e treinamento, onde $30 \%$ dos dados serão de teste. Então treinamos os $70 \%$ com cada um dos algoritmos. A Tabela 3 
mostra o resultado arredondado dos algoritmos no conjunto de dados de teste.

TABELA 2. RESULTADO DOS ALGORITMOS

\begin{tabular}{|c|c|}
\hline ALGORITMO & ACURÁCIA TESTE \\
\hline Regressão Logística & $73 \%$ \\
\hline Random Forest & $56 \%$ \\
\hline Naive Bayes & $60 \%$ \\
\hline Multilayer Perceptron & $60 \%$ \\
\hline KNN & $53 \%$ \\
\hline
\end{tabular}

Comparando os resultados da acurácia dos algoritmos, foi constatado que o classificador que apresentou o melhor resultado para o dataset OASIS-1 foi a regressão logística. Durante o teste constatou-se que dos 38 casos em que o CDR é igual a zero, o algoritmo classificou corretamente 32 deles.

Os resultados anteriores foram utilizando os hiperparâmetros padrão dos algoritmos. Com o objetivo de obtermos melhores resultados, selecionamos alguns algoritmos para alteramos esses hiperparâmetros. Foram eles, Random Forest, Multilayer Perceptron e KNN.

Alterando a profundidade da árvore para $6 \mathrm{e}$ o random number para 10, foi possível aumentar a acurácia do algoritmo Random Forest. Determinamos que outras combinações dos atributos não melhoraram sua performance, tendo em alguns casos até a diminuído.

Quanto ao algoritmo Multilayer Perceptron, apesar de alterarmos o número de camadas ocultas, não conseguimos melhorar o resultado obtido anteriormente. Independente da combinação que fizéssemos, o resultado era o mesmo ou pior.

Por fim, ao aumentarmos o número $\mathrm{k}$, ou seja, o número de vizinhos no algoritmo $\mathrm{KNN}$, obtivemos um resultado melhor que o anterior. A Tabela 4 apresenta os novos resultados.

TABELA 3. RESULTADOS DOS ALGORITMOS APÓS ALTERAÇÃO DOS HIPERPARÂMETROS

\begin{tabular}{|c|c|}
\hline ALGORITMO & ACURÁCIA TESTE \\
\hline Random Forest & $64.3 \%$ \\
\hline Multilayer Perceptron & $60 \%$ \\
\hline KNN & $61 \%$ \\
\hline
\end{tabular}

Observamos que, apesar das alterações feitas nos hiperparâmetros, não foi possível alcançar uma acurácia melhor do que a obtida com o algoritmo de Regressão Logística, sendo este o modelo escolhido para o problema.

\section{CONCLUSÃO}

Esse artigo teve como objetivo agregar aos estudos de prevenção a demência e a doença de Alzheimer. Atualmente, a demência é um grande problema de saúde global, pois não tem cura. O grande foco é na redução de riscos, intervenção precoce e diagnóstico oportuno da doença em idosos. Conforme observado nas pesquisas da literatura, hoje em dia há estudos científicos voltados para o mesmo assunto, mas com diversos focos utilizado dados e métodos que não tínhamos anteriormente.

Há estudos sobre detecção precoce de demência nos indivíduos usando Machine Learning e inteligência artificial, no entanto, ainda há a necessidade de identificar os atributos relevantes que podem detectar a demência em um estágio muito inicial. No presente trabalho utilizamos a idade, o nível socioeconômico e nível de escolaridade para a construção do modelo, mas para pesquisas futuras seria interessante considerar outros atributos, pois é possível que com outros, a acurácia na classificação do nível do declínio cognitivo seja maior.

Apesar da regressão logística ter apresentado uma acurácia de $73 \%$ e o ideal seria um valor maior, pudemos avaliar o quanto atributos tão comuns como os já citados, podem influenciar a saúde mental do indivíduo. Além disso, também constatamos que as determinadas características estão interligadas, como o nível socioeconômico e o nível educacional; idade e tamanho do cérebro; tamanho do cérebro saúde mental; nível educacional e declínio cognitivo.

Futuramente, além de utilizar outros atributos para a análise, como o resultado do mini exame do estado mental, também devemos, idealmente, usar uma base de dados maior. A que utilizamos não é considerada grande para o treinamento de algoritmos de Machine Learning, ainda mais com tantas informações faltantes. Assim, profissionais da área da saúde poderão utilizar este e novos estudos para identificar com maior rapidez e exatidão a doença ou seu possível desenvolvimento, podendo tratá-la antes de apresentar seus primeiros sintomas e assim sendo capaz de proporcionar uma melhor qualidade de vida para o idoso e sua família.

\section{REFERÊNCIAS}

[1] ORGANIZAÇÃO MUNDIAL DE SAÚDE, "Dementia". Disponível em: $<$ http://www.who.int/mediacentre/factsheets/fs362/en/>. Acesso em: 10 Julho. 2020.

[2] CASOS DE DEMÊNCIA VÃO TRIPLICAR E CHEGAR A 152 MILHÕES DE PESSOAS ATÉ 2050. Jornal Globo, Rio de Janeiro, 14 de maio de 2019. Disponível em: <https://g1.globo.com/ciencia-esaude/noticia/2019/05/14/casos-de-demencia-vao-triplicar-e-chegar-a152-milhoes-de-pessoas-ate-2050-diz-oms.ghtml>. Acesso em: 10 Julho 2020 .

[3] O QUE É A DOENÇA DE ALZHEIMER? Disponível em: $<$ http://www.alzheimermed.com.br/perguntas-e-respostas/o-que-causaa-doenca-de-alzheimer $>$. Acesso em: 15 agosto 2020.

[4] CASOS DE DEMÊNCIA VÃO TRIPLICAR E CHEGAR A 152 MILHÕES DE PESSOAS ATÉ 2050. Disponível em: $<$ https://neuroforma.com.br $>$. Acesso em: 15 agosto 2020.

[5] WHAT IS DEMENTIA? Disponível em: $<$ https://www.alz.org/alzheimers-dementia/what-is-dementia $>$. Acesso em: 10 out. 2020

[6] DOENÇA DE ALZHEIMER. Disponível em: $<$ http://bvsms.saude.gov.br/dicas-em-saude/2056-doenca-de-alzheimer>. Acesso em: 15 agosto 2020.

[7] OPEN ACCESS SERIES OF IMAGING STUDIES (OASIS). Disponível em: <http://www.oasis-brains.org/>. Acesso em: 10 junho 2020.

[8] A DEEP LEARNING MODEL TO PREDICT A DIAGNOSIS OF ALZHEIMER DISEASE BY USING 18F-FDG PET OF THE BRAIN. Disponível em: $<$ https://pubs.rsna.org/doi/pdf/10.1148/radiol.2018180958>. Acesso em: 20 setembro 2020.

[9] Frank J. Wolters, Hazel I. Zonneveld, Silvan Licher, Lotte G.M. Cremers, on behalf of the Heart Brain Connection Collaborative Research Group, 
M. Kamran Ikram, Peter J. Koudstaal, Meike W. Vernooij, M. Arfan Ikram. Neurology Aug 2019, 93 (9) e917-e926. HEMOGLOBIN AND ANEMIA IN RELATION TO DEMENTIA RISK AND ACCOMPANYING CHANGES ON BRAIN MRI. Disponível em: $<$ https://n.neurology.org/content/93/9/e917>. Acesso em: 20 setembro 2020.

[10] HEMOGLOBINA.

Disponível

em: $<$ https://www.biologianet.com/biologia-celular/hemoglobina.htm>. Acesso em: 20 setembro 2020.

[11] MEMORY LOSS REVERSED OR ABATED IN THOSE WITH COGNITIVE DECLINE. ScienceDaily. ScienceDaily, 31 July 2020. Disponível em: <www.sciencedaily.com/releases/2020/07/200731104124.htm>. Acesso em: 31 Julho 2020.

[12] MACHINE LEARNING: O QUE É? E POR QUE É TÃO IMPORTANTE? Disponível em: < https://navita.com.br/blog/machinelearning-o-que-e-por-que-e-tao-importante/>. Acesso em: 31 Julho 2020.

[13] MACHINE LEARNING: O QUE É, CONCEITO E DEFINIÇÃO. Disponível em: <https://www.cetax.com.br/blog/machine-learning/>. Acesso em: 20 janeiro 2021.

[14] MEHMET KOCAK, MD, RUSH UNIVERSITY MEDICAL CENTER. RESSONÂNCIA MAGNÉTICA. Disponível em: $<$ https://www.msdmanuals.com/pt/profissional/ressonancia-magnetica $>$. Acesso em: 31 outubro 2020 .
[15] REGRESSÃO LOGÍSTICA. Disponível em: $<$ https://pt.wikipedia.org/wiki/Regressao_logistica>. Acesso em: 18 dezembro 2020.

[16] APRENDENDO EM UMA FLORESTA ALEATÓRIA. Disponível em: $<$ https://medium.com/machina-sapiens/o-algoritmo-da-florestaaleat $\%$ C3\%B3ria-3545f6babdf8>. Acesso em: 08 fevereiro 2021.

[17] ÁRVORES DE DECISÃO. Disponível em: < https://minerandodados.com.br/arvores-de-decisao-conceitos-eaplicacoes/>. Acesso em: 15 fevereiro 2021.

[18] COMO FUNCIONA O ALGORITMO NAIVE BAYES. Disponível em: $<$ https://minerandodados.com.br/naive-bayes-machine-learning/> Acesso em: 09 fevereiro 2021.

[19] REDE NEURAL PERCEPTRON MULTICAMADAS. Disponível em: $<$ https://medium.com/ensina-ai/rede-neural-perceptron-multicamadas. $>$ Acesso em: 15 fevereiro 2021.

[20] REDES NEURAIS, PERCEPTRON MULTICAMADAS E O ALGORITMO BACKPROPAGATION. Disponível em: < https://medium.com/ensina-ai/redes-neurais-perceptron-multicamadas-eo-algoritmo-backpropagation-eaf89778f5b8>. Acesso em: 16 fevereiro 2021.

[21] MACHINE LEARNING NA PRÁTICA COM O ALGORITMO KNN. Disponível em: <https://minerandodados.com.br/machine-learning-napratica-knn-python/>. Acesso em: 15 fevereiro 2021. 